\title{
Neck Dissection Impairment Index
}

National Cancer Institute

\section{Source}

National Cancer Institute. Neck Dissection Impairment Index. NCI Thesaurus. Code C107226.

A health-related instrument to identify the factors that affect quality of life related to shoulder dysfunction following neck dissection for head and neck cancers. 